\title{
Autonomous Meridian Sensory Response - From Internet Subculture to Audiovisual Therapy
}

\author{
Diego Garro \\ Keele University \\ Music and Music Technology \\ The Clockhouse, Keele University \\ Staffordshire ST5 5BG, UK \\ d.garro@keele.ac.uk
}

\begin{abstract}
ASMR (Autonomous Sensory Meridian Response) is the name given to a pleasant sensation that can be felt most commonly on the scalp and can be triggered by various gentle sounds (like whispers, crinkles or tapping), smooth and repetitive visual stimuli, personal attention (like the touch of a hairdresser or a masseur) or other events. ASMR is often associated with a general feeling of relaxation and peace. Whilst academic research on the sociological, artistic, sensory and cognitive dimensions is still in its infancy ASMR has grown into a worldwide, cross-disciplinary, inter-cultural, multi-lingual social media sensation. This paper outlines the rise of ASMR as Internet subculture from its inception as 'whispering community' on Internet platforms and blogs, to become a truly popular (i.e., made by the people) platform for creative expression, self-made holistic therapy and, in some instances, true artistic audiovisual endeavours.
\end{abstract}

This paper comments on the reasons behind the rise of the ASMR community as a fertile ground for creative expression. Audiences' expectations are dictated by the attention-induced nature of the sensory experience, a factor that spawned an exceptionally perceptive viewership if one considers the inherently fragmented essence of ubiquitous streaming media and the impatient scanning and skipping modes of reception it encourages. 'ASMRtists' thus enjoy a privileged relationship with audiences who are not impressed with the relentless pour of energy and information from social media platforms and treasure, instead, the slow, the quiet, and the subtle.

Examples from various ASMR content creators will be analysed from the compositional standpoint, highlighting technical and idiomatic similarities with forms of improvisatory practices and experimental artistic languages such as Musique Concrète. The paper will also illustrate recent audiovisual projects related to ASMR carried out at Keele University and will introduce the audience to planned developments towards ASMR related content delivered through mobile platforms.

ASMR. Autonomous. Sensory. Meridian. Response. Whispering. Community. YouTube. Tingling. Tigles. Sensation. ASMRtist. ASMRer. Creator. Chills. Frisson. Healing. Therapy. Musique. Concrète. Electroacoustic. Anecdotal. Composition.

\section{INTRODUCTION}

There is a quiet, no, a very quite storm across the Internet. It is caused by a solitary individual in a garden shed or in a little study in the corner of the house, the room furthest away from the noise of the main street. Pointed towards him/her you would see a camera as well as one or more microphones of the highest quality. He/she is performing a strange ritual made of gentle sounds, whispers and hand movements. It is filmed close-up. The sound and the vocal utterances, barely audible, are recorded rigorously in stereophonic, often binaural (MassageASMR 2015a). On YouTube, more than a hundred thousand people around the world will watch and listen to that audio-video recording, placated, mesmerised, engrossed, like an audience at a concert of a lifetime. What brings our actor and audience together is a shared experience of a curious tingling sensation in the scalp, triggered by the particular visual and sonic events featured in the recording. It is also the shared experience that 
shaped an on-line community which started by pure chance as a result of a somewhat bizarre thread on a health-related blog (Richards 2017) initiated by username okaywhatever 51838. The first 'whispering' YouTube channel started in 2009 and the first video uploaded by username WhisperingLife was nothing more than an experiment (WhisperingLife 2009), just short of two minutes long, on broadcasting a recording of a whispering voice with no video track as such. The view count for such video Whisper 1 - Hello! is 130,720 at the time of writing. The WhisperingLife YouTube channel has now more than 1.2 million views in total.

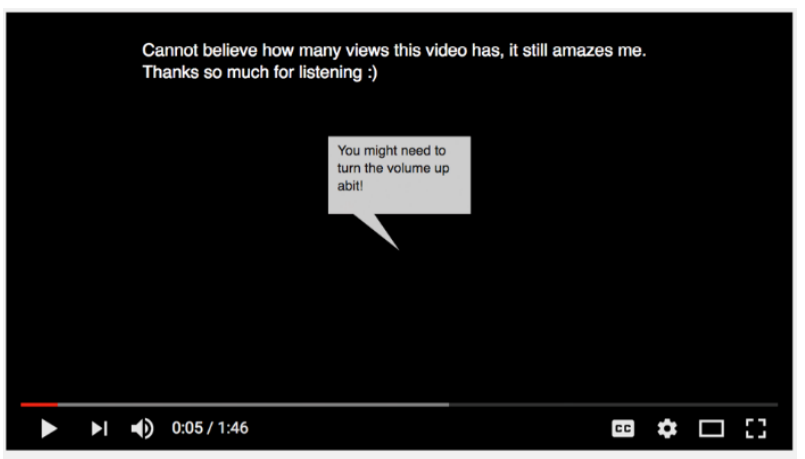

Whisper 1 - hello!

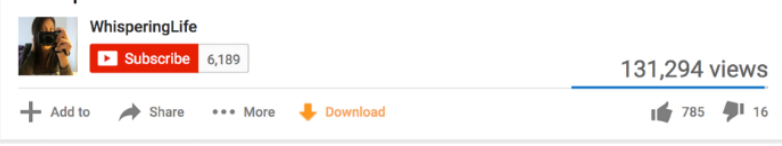

Figure 1: The first ASMR video. WhisperingLife's 'Whisper 1 - hello!'

The whispering community harnessed the power of social media and rapidly gathered growing numbers of followers, readers and bloggers who debated on the causes of the scalp-tingles and on the experiences that elicited them. Content creators provided audio-video material suitable to trigger the effect. In the early years of the on-line community the names given to the sensorial condition changed, depending on what blog and what thread one followed: it was the 'weird tingling sensation', at first; then it was named the 'Attention Induced Head Orgasm (AlHO)', then the 'Attention Induced Observant Euphoria', and eventually 'Autonomous Sensory Meridian Response' (ASMR). Jennifer Allen, the founder of the first Facebook group dedicated to ASMR-related topics (Autonomous Sensory Meridian Response Group 2012) coined the term in 2010.

Whilst ASMR continued its exponential growth as an on-line community, the attention of the scientific world was limited, and unsurprisingly cautious due to the ephemeral and quintessentially individual nature of the experience to be examined.

In 2015, a full search on EBSCO with keywords ASMR .or. AlHO .or. Attention Induced Observant
Euphoria returned no peer-reviewed academic article on the matter. In March 2017, the same search returned a few articles centered on the neurology and the sociology of ASMR.

Early studies (Novella 2012) posited the similarity between ASMR episodes and seizures. The first study of the ASMR condition provided quantitative analysis of the 'triggers' as well as an examination of the effects on mood and on the relationship with flow-state (Barratt and Davis 2015). It also linked ASMR to conditions such as synaesthesia and misphonia.

The neurological aspect of the scalp-tingling condition has been investigated with great difficulty. Measuring subjects' responses can be problematic using the type of invasive techniques (MRI scanner, EEG sensor) that inevitably hinder the uniquely contradictory physical and mental state (relaxation, silence, combined with focus, concentration) under which ASMR episodes occur most often (Smith, Fredborg \& Kornelsen 2016). In this study the authors linked the ASMR condition to particular patterns of the Default Mode Network, the system of interacting bran regions that activates during states of rest and mind-wandering. Subjects who experience ASMR show a higher-thanaverage ability to activate multiple resting modes and lower their inhibition towards emotional experiences while doing so. In other words, ASMR seems to be linked with particular brain patterns, and emotional openness in situations of contemplative rest. This study does not provide an explanation as to why ASMR manifests itself with the particular tingling sensations emanating from the scalp. Novella's contention (the mini-seizures theory) provides at least a hypothetical explanation of the physical manifestation of ASMR. Conversely, Smith, Beverley and Kornelsen's study examines the brain neurological underpinnings of ASMR. However, an integrated theory linking the two aspects is yet to be developed.

An investigation into the psychological dimension of ASMR has begun only very recently and some preliminary results have indicated a correlation between predisposition to ASMR and certain personality traits (Fredborg, Clark \& Smith 2017).

From the viewpoint of Media and Cultural studies, ASMR can be examined as a form of mediated communication in which a form of remote intimacy is established through the mechanisms of psychological affect (Andersen 2015). In her study Andersen discusses the nature of the ASMR online community and its problematic relationship with notions of closeness, pleasure, sexual experience and normative notions of gendered care and sexuality. 
Criticism of ASMR as a body of audiovisual broadcasts focuses on the novel intercommunication language established by ASMR practices (Gallagher 2016). Gallagher explores, in particular, the socio-political potential of the dialogue established between creators and users, which valence is particularly noteworthy in the frantic, congested medium of the Internet.

In response to its growing popularity ASMR has also attracted the attention of mainstream media (Higham 2014). Unsurprisingly, such scrutiny has focused, in several occasions, on the inherently queer aspects of ASMR culture as seen from the angle of uninitiated observers and commentators. Often in a derisive manner, the narrative of ASMR as a proxy for online sexual intimacy has obscured a deeper analysis of its roots and of the inner values shared by the members of this fascinating community.

In this paper I will focus on some of the aspects that have given ASMR a unique place in contemporary Internet culture as a forum for popular creative endeavours and for their appreciation as a mean to various ends. These include both the experience of the ASMR tingling sensation, and more in general, and perhaps more importantly, the experience of audio-visual constructs as a mean towards relaxation, sleep and general psychological wellbeing.

\section{THE MICROPHONE AS A NEW/OLD TOTEM}

Initial examples of ASMR videos were rather crude home recordings of whispered tales or ramblings captured using consumer level technology, most likely cheap USB microphones or even the built-in microphones available on laptop computers. From these early steps, the whispering community quickly realised that 'trigger sounds' (i.e., the events capable of eliciting the ASMR reaction) were better appreciated in particular recording and listening conditions. Most of the popular ASMRtists developed a more sophisticated 'idiom', both visually and (especially) sonically. With higher demands on content and quality they approached sound capture not much differently from sound designers and electroacoustic composers. The microphone is at the heart of 'ASMRtistry'. It functions not only as an acoustic-electric transducer and recording device; it is utilised as the sonic equivalent of a magnifying glass, a vehicle to the inner fabric of the subtle sound events that are cornerstones of the ASMR language: whispers, unintelligible vocal sounds and artefacts that are part of speech activity (lip and tongue sound, breathing), ruffles of fabric, voluntary and involuntary tapping and scratching, etc. Close-up and even closer is the imperative here. We hear heightened versions of these common sounds in fashions that conjure up notions of proximity between me (listener) and him/her (the maker) hence, inevitably rouse ideas of intimacy.

Thus, many ASMR content creators invested in expensive microphone setups focussing on specifications such as sensitivity, low self-noise and stereophonic capability. The first two are concerned with the demands presented by recordings of subtle noises characterised by low values of sound pressure level, for example a single crinkle of a gently bent plastic wrap. We witness, here, a shift from sonic closeness to sonic purity and fidelity.

The concern with stereophony is linked to the triggering potential attributed by many 'ASMRers' to sounds distinctly localised on either side of the leftright sound field. The three-dimensional effect created by binaural recordings listened through headphones or earphones also serves the purpose of creating and conveying an immersive sound world where the contrast between proximity and distance is intensified, yet another pillar of the ASMR language.

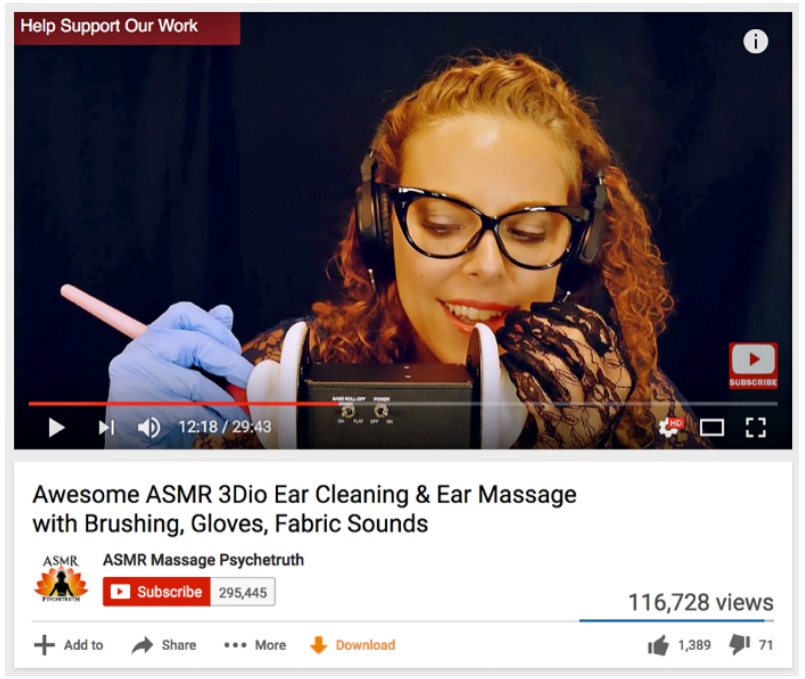

Figure 2: ASMR Massage Psychetruth using the popular 3Dio Free Space Binaural Microphone

The microphone fetish in ASMR culture manifests itself both in the attention to recording quality, but also in the desire to share with the community facts, tools and techniques related to ASMR audio capturing (Tony Bomboni ASMR 2016).

The primacy of the microphone in ASMR culture has undoubtedly acquainted a large population of non-specialists to the power of this instrument and the windows it opens to those who have an interest in exploiting the sense of attentive hearing, nowadays often neglected or violated. It can be argued that, in just a few years ASMR culture has done more for the sound-art world, and arguably for acoustic ecology, than decades of avant-garde 
experimentalism, government funded projects and active listening education in schools and Universities. It would certainly be naïve to assume that the millions who watch ASMR videos on YouTube are ready to engage with erudite forms of sonic (and video) art, but perhaps it is worth considering whether ASMR itself has already consolidated itself as an endogenous artistic language focussed on the primacy of the ear, with its rituals and idiosyncrasies. As such, many ASMR video recordings may be construed as a modern audiovisual manifestations of anecdotal electroacoustic music (Böhme-Mehner 2012) that is the sub-genre concerned with the artistic, often playful or whimsical, use of recognisable microphone recordings, more or less manipulated, linked to specific objects, places, contexts or memories.

\section{A DOMESTIC PARLOUR, RELOADED}

The need for quiet, silent settings for the enjoyment of ASMR audio-video content has rekindled an interest in suitable domestic spaces for creative media playback, after the historical tides of $\mathrm{Hi}-\mathrm{Fi}$ systems, in 1950s-1990s, and advanced HomeTheatre, in the 2000s. Many ASMRers report that lying in bed constitutes their favourite playback location, but sitting at home in a peaceful room in front of a computer or a laptop are also popular solutions (ASMR sub-reddit 2014a). In an age of smart-phones and mobile listening, ASMR has brought viewers back into the house, searching for 'listening pods', where a good degree of control can be regained over the sonic environment, against increasingly invasive urban soundscapes (Atkinson 2008). It also renewed the politics of gendered domestic spaces originally linked to the advent of domestic Hi-Fi systems. Whereas the Hi-Fi, with its technological masculine connotations, put men in control of the domestic media platforms (Keightley 1996) ASMR, which is quintessentially an individual occupation, seems to have zeroed this gender gap in terms of domestic technological platform. If anything, ASMR has extended to all members of a household the typical teenager preoccupation with the bedroom as solitary sanctuary (Atkinson 2008).

If the practice of ASMR content creation is dominated by the microphone, as a cultural and artistic construct, the domestic ASMR pod is conversely focussed around the headphones as a mean to both insulation from a potentially intrusive soundscape and a vehicle to the appreciation of sonic detail and spatial, even binaural, cues in the recordings. The utilitarian use of ASMR videos for relaxation and sleep has boosted an interest in flatline, comfortable headphones sets, which allow user to sleep comfortably while wearing them. At the fringe of the ASMR community, there are also attempts at kitting the bedroom (and the bed itself) with loudspeakers and media playback setups specifically designed for the headphones-free enjoyment of ASMR content, as well as anything else conducive to someone's sleep (ASMR subreddit $2014 b$ ).

ASMRers have thus engaged in a quest for a rediscovery of a new, individual domestic parlour, which for many seems to acquire the meaning and function of a spa within their own household and, for some, becomes a true place of solace away from personal troubles or psychological distress.

\section{THE MANIPULATION OF TIME THROUGH OTHER MEANS}

Many ASMR viewers seek the pleasurable tingling sensation triggered by the sounds and images provided, largely for free, by content creators across Internet platforms, especially YouTube. In fact, the influential content aggregator Reddit ranks ASMR videos on the basis of their success as tingling triggers as reported by users (Gallagher 2016). The content of many ASMR videos is, despite its utilitarian purpose, rather trivial, at least on first looking: we see close-ups of the artist whispering, handling some noise-making objects, acting out quaint role-play procedures. ASMRtists themselves almost invariably feature in their own self-made videos, sometimes wearing costumes and make up, impersonating personal care providers, like a friend, a hairdresser, a doctor. Thus, the fast growing ASMR community have the hallmarks of an on-line fair of the ephemeral, dedicated to nothing other than unapologetic escapism. In truth, it has indeed been portrayed as such in many of the early covering features in mainstream media. However, for a less superficial understanding of the ASMR phenomenon we need to step inside the circle and unravel the very distinctive type of communication in place between the many-thousand individual artist and the millions individual viewers who watch their videos.

In many forums, surveys and social network discussion threads, viewers report and debate as much on the scalp-tingling response as they do on the relaxing effect of the ASMR videos they regularly access. The language of ASMR is somewhat eccentric; it features events which unfold at much slower pace than any other audio-video content currently available on any other media (TV drama, films, video-games, news). Indeed, ASMR is much more sedated than our busy working patterns, our hectic daily travels. Fundamentally, it is much, much slower than the life most of us live. Hence, we are witnessing a very large and fastgrowing population of onlookers all seeking for a similar experience: the slowing down of their time. 
Not the chronometric time, obviously, as that is a feat of science (fiction?). It is the perceived expanding of the experiential time that ASMRers seem to strive for, and ASMRtists so skilfully provide. Therefore, what may be read as an escape into the ephemeral may conceal a subtler, and somewhat revolutionary, desire to regain control over someone's time, the time that our modern life increasingly, relentlessly takes away from us.

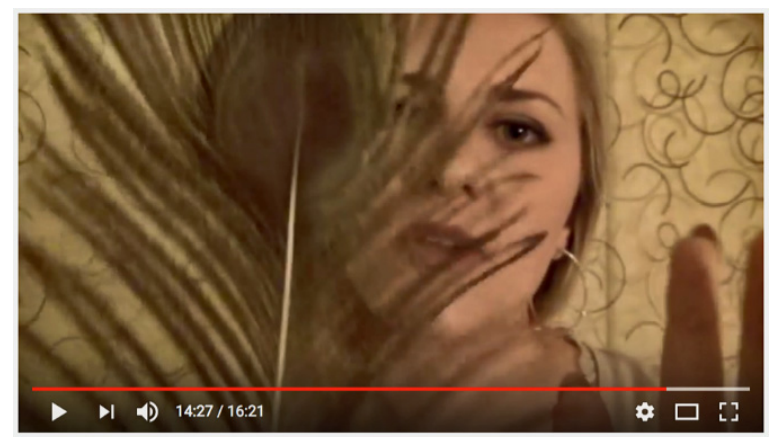

*_* Oh such a good 3D-sound ASMR video *_*

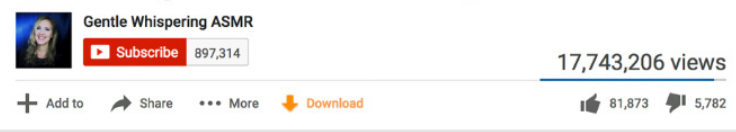

Figure 3: Gentle Whispering ASMR most popular ASMR video to date.

As I watch MassageASMR's twenty hours of tapping sounds for sleep and relaxation (MassageASMR 2015b) I gain a new perspective on the passing of time and my place in it.

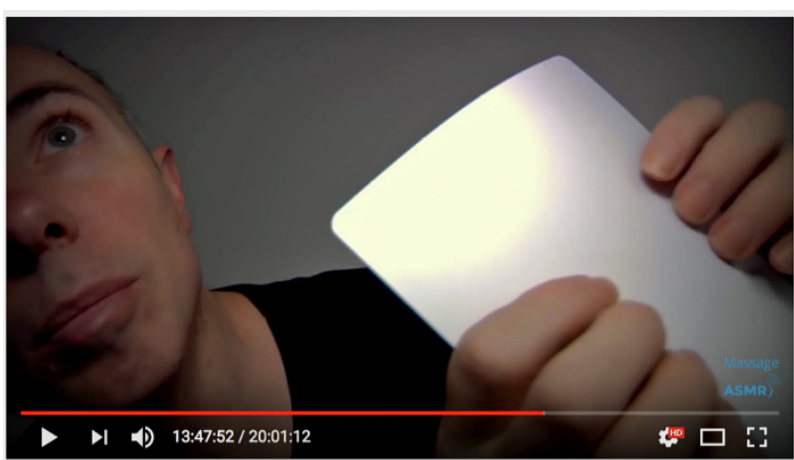

ASMR 20 Hours of Tapping Sounds for Sleep \& Relaxation

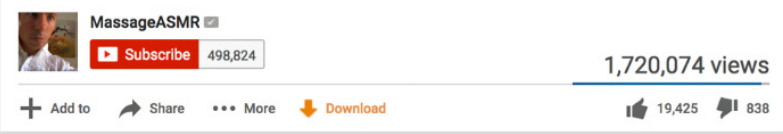

Figure 4: Stretching time: MassageASMR's 'twenty hours of tapping sounds for sleep and relaxation'

This is helped by the relationship between the sound events and the mesmerising power of Dmitri's (the author) assured and relaxed body language, which delivers repeated patterns of motions akin to a musical ostinato. After the first few seconds, we know what type of sounds he is going to create and how. We expect them and we see them coming; however, the subtle variations in the timing of execution create a spellbinding tension. It is the audiovisual equivalent of an expressive rubato, hence quintessentially musical.

The compositional / improvisational skills of ASMRists may be instinctive but they are nevertheless responsible not only for the success of their work as triggers, but also as time-bending artefact. In ランプシェードマン (Lampshade Man) / ASMR performance (Ephemeral Rift 2015), the artist conjures up a very slow, yet enthralling video flirting with the acousmatic paradigm of much electroacoustic music, whereby we hear the sounds but are not able to see the sources of all of them (in this case the lampshade covering his head hides the source of the whispered vocalisations). The mise-en-scène of Lampshade Man implies a reverberant indoor setting, yet we hear extremely 'dry' sound thanks to close-up microphone recording taking place under the shade. Like most ASMR videos Lampshade Man is filmed and recorded in one long take but the improvisation is clearly organised in separate sections, alluding to some kind of form in lieu of a filmic narrative, which clearly is absent.

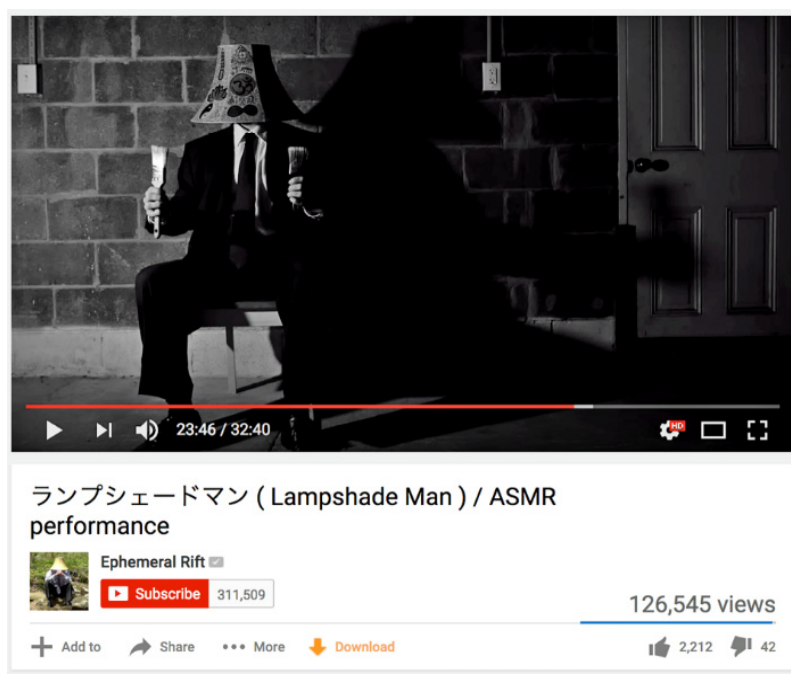

Figure 5: Stretching time: Ephemeral Rift's 'Lampshade Man' / ASMR performance

This hypnotic adagio features mostly individual sound sources, another common feature of ASMR sonic orchestration which facilitates the discernment of long, evolving events and the type of time-mind-wandering typically associated with ASMR.

\section{HEALERS, HEALERS, AND MORE HEALERS}

Surveys of viewers' background, motivations and habits revealed that ASMR content is often utilised as vehicle to relaxation and as a coping strategy 
against anxiety. In fact, ASMR-style audio recordings are marketed, and sold, as therapy aids for relaxation (Gracetone 2012), indicating that the triggering of the tingling sensation is not necessarily the only, or not even the main, goal sought by ASMR viewers. This is not surprising considering the traits of ASMR productions in terms of content, idiom and temporal organisation of the events (see previous section). The peculiar role of the ASMRtist as a close-up narrator and performer has a powerful influence in the relationship between him/her and the viewer. Although this is a mediated, remote influence, it is nevertheless very real for both artists and audiences. The actor/artist on the screen often behaves (or acts) calmly, confidently and caringly. The atmosphere of closeness and intimacy thus generated seem to have a potent effect on many individuals who seek strategies to cope with stress, anxiety, pain, depression and insomnia (Barratt \& Davis 2015). There is no exchange and administration of drugs here (hence no side effects); just the interaction, mediated through an Internet platform, with charismatic individuals who perform a variety of noise-making activities on the screen, mostly in a very measured, gentle fashion. The mechanisms at play during such encounters are clearly psychological or even psychosomatic, and can be construed as a modern equivalent of the interaction with, and effect of, healers. ASMRtists' reverence for the viewers and viewers' trust in the artists' genuine intents are the very same pillars of an effective interaction (in terms of recovery form an illness) between patients and health care providers (Hanvey 2007).

Loneliness is one of many epidemics of our modern times. It is compelled by the lengthening of our life expectancy and the rise of an elderly population left at the fringes of a collapsing social care system. It affects many young people too, caught between competing demands of study, financial hardship and career. In these contexts, an individual who is confronted with issues of stress, insomnia or anxiety may find themselves for the first time at the centre of a carer's attention when they place themselves in the hand of health professionals. Even subjects who do not necessarily hold a specific faith in the power and efficacy of spiritual or religious healing may still reckon mental health practitioners spiritual healers (Stott 2010).

The therapeutic dimension of ASMR empowers both artists', who adopt the healer role without specific medical or religious training, and viewers, who have a considerable level of control over the choice of 'healing content', the timing and the context where the amelioration takes place. The staggering number of motivational messages more or less explicitly embedded in ASMR content seem to confirm many artists' awareness of a widespread desire for healing and progress, and attest to their ambition to fulfil it. Even in the middle of a, raher comical, role-play based on an improbable imaginary Illuminati doctor figure, artist TirarADeguello manages to convey very deep and fervent messages of personal empowerment and secular humanisms (TirarADeguello 2017).

Healing is sometimes the thematic reference and/or intent of ASMR videos. Yu will find entire channels dedicated to healing (Cooper 2017). You will also find artists engaging in healing role plays as part of one or more of their videos (WhispersRed ASMR 2015). A rigorous foundation for the healing function of ASMR is just as problematic, if not more problematic, as the scientific support for any healing practices. Nevertheless, the therapeutic element of ASMR is, for whatever reason, a very prominent aspect of the shared system of values, expectations and achievements of this online community. It is reported to work by several of the viewers (Barratt \& Davis 2015); it is possible that it works purely as a placebo, but it works nonetheless.

\section{ASMR AND THE ARTS OF QUIET}

From within the mainstream of ASMR culture, as well as from its fringes, a breed of audio-video artists/broadcasters have connected directly, unfiltered, with a substantial online community without the mediating role of institutions, art galleries, labels, distributors. This is a captivating example of popular broadcasting; popular in the sense that is made by untrained enthusiasts with relatively limited financial and technical means, for a widespread audience. Using ASMR culture as a departing lounge, content creators of all ages and provenances have utilised this idiom as a platform for a variety of artistic expressions and cultural endeavours: comedy (Ephemeral Rift 2011), literature and creative writing (Phoenician Sailor 2014), history and science (The French Whisperer 2013), painting (Brad Robbo 2006), music (Infrasonge 2015), sound art (ASMR By Design 2012), drama (Miniyu ASMR 2016).

The expression of so much inventiveness is perhaps the most endearing legacy of ASMR and a vindication against stereotypes of ASMR as a vehicle for sexualised content fuelled by several videos that carries an 'ASMR' tag.

While the international community of ASMRtists is still growing, some of its most successful representatives are pushing the boundaries of what can be achieved with this form of communication. They are doing so harnessing the opportunities offered by Virtual Reality and interactive technology (PixelWhipt 2015). 


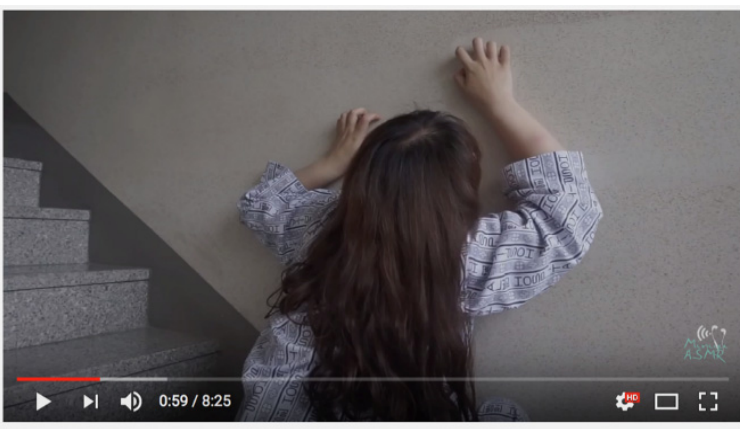

[Miniyu's Drama ASMR] Still Waiting (Role play)

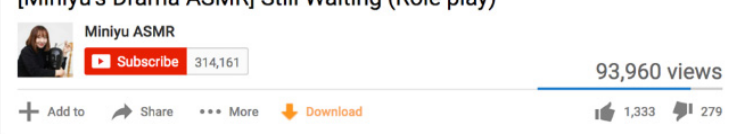

Figure 6: Drama and ASMR. Miniyu ASMR's 'Still Waiting (Role play)'.

The selection of content mentioned in this paper illustrates the vitality of the ASMR culture and the inherent openness towards novelty. It is a language that has developed quickly and branched out into many different directions, while still maintaining common idiomatic traits.

The other area of growth is the demography of the ASMR community, which seems to be expanding at steady rate. Anecdotal evidence seems to suggest a certain bias towards USA and UK in the population of content creators and viewers. However, there is no doubt that the ASMR community is increasingly international, and intergenerational, with ASMRtists as young as ten (Taylor Robbins 2016).
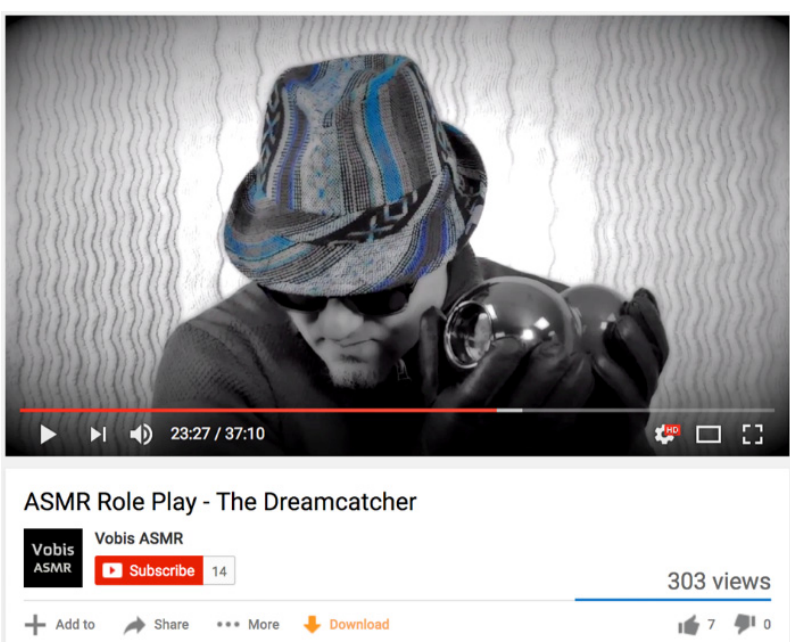

Figure 7: Pilot study on ASMR / Video Art hybridisation. Vobis ASMR, 'The Dreamcatcher'.

A forthcoming research project at Keele University (UK) will investigate the use of mobile applications to deliver interactive audiovisual compositions in the style of ASMR. This project follows recent analytical and practical engagement with the ASMR culture (Vobis ASMR 2016) which has highlighted technical and idiomatic similarities with forms of improvisatory practices and experimental artistic languages such as Musique Concrète and Visual Music.

The drive behind these projects is to expand further the possibilities offered by a cultural framework that has already demonstrated a remarkable flexibility and an astonishing potential for a variety of mediated communications, artistic expression and wellbeing.

\section{REFERENCES}

Andersen, J (2015) Now You've Got the Shiveries: Affect, Intimacy, and the ASMR Whisper Community. Television \& New Media, 16(8), pp. 683-700.

ASMR By Design (2012) YouTube channel. https://www.youtube.com/user/ASMRByDesign/feat ured (retrieved 19 March 2017).

ASMR sub-reddit (2014a) How do you watch your asmr Videos?

https://www.reddit.com/r/asmr/comments/1vi31w/h ow do you watch your asmr videos meta/

ASMR sub-reddit (2014b) ASMR Setups for Bedtime?

https://www.reddit.com/r/asmr/comments/1p648b/m eta asmr setups for bedtime/

Atkinson, R (2008) The Home as Aural Haven. Lo sQuaderno, 10(1), pp.5-11.

\section{Autonomous Sensory Meridian Response Group (2012)}

https://www.facebook.com/groups/ASMRGroup/ (retrieved 19 March 2017).

Barratt, E.L. and Davis, N.J. (2015) Autonomous Sensory Meridian Response (ASMR): a flow-like mental state. PeerJ. DOI: $10.7717 /$ peeri.851

Böhme-Mehner, T. (2012) Between Meaning and Meaningfulness - "Understanding" Anecdotal Music. Proceedings of the Electroacoustic Music Studies Network Conference, Meaning and Meaningfulness in Electroacoustic Music, Stockholm.

http://www.ems-network.org/spip.php?article333 (retrieved 19 March 2017).

Brad Robbo (2006) YouTube channel. https://www.youtube.com/user/BraDRoBBo/feature d (retrieved 19 March 2017).

Ephemeral Rift (2011) YouTube channel. https://www.youtube.com/user/EphemeralRift (retrieved 19 March 2017). 
Ephemeral Rift (2015) ランプシェードマン ( Lampshade Man ) / ASMR. https://www.youtube.com/watch?v=nTAVUUXaFG $\underline{\text { M }}$ (retrieved 19 March 2017).

Fredborg, B., Clark, J., and Smith, S. D. (2017) An Examination of Personality Traits Associated with Autonomous Sensory Meridian Response (ASMR). Frontiers in Psychology, 8: 247. DOI: 10.3389/fpsyg.2017.00247

Gallagher, R. (2016) Eliciting Euphoria Online: The Aesthetics of "ASMR" Video Cult. Film Criticism, 40(2), pp. D1-D15.

Gracetone (label) (2012) Relaxation Therapy (Soft Spoken Whisper-Asmr Stress Relieving Therapy). https://www.amazon.co.uk/Relaxation-TherapySpoken-Whisper-Asmr-Relieving/dp/B0086RIR2M (retrieved 19 March 2017).

Hanvey, J. (2007) Who is this patient? Southern Medical Journal, 100(1), pp.91-95.

Higham, N. (2014) ASMR: The videos which claim to make their viewers 'tingle'. BBC.

http://www.bbc.co.uk/news/magazine-30412358

(retrieved 19 March 2017).

Infrasonge ASMR \& Soundscapes (2015) YouTube channel.

https://www.youtube.com/channel/UCoLVOyJbpTK SUvvCQPaeuNw/featured

(retrieved 19 March 2017).

Keightley, K. (1996) 'Turn it down!' she shrieked: gender, domestic space, and high fidelity, 1948-59. Popular Music, 15(1), pp.149-177.

MassageASMR (2015a) ASMR Soft Paper, Crinkle and Almost Inaudible.

https://www.youtube.com/watch?v=CZv5DpnS-P8 (retrieved 19 March 2017).

MassageASMR (2015b) ASMR 20 Hours of Tapping Sounds for Sleep \& Relaxation. https://www.youtube.com/watch?v=wya8Qd5Z7y4 (retrieved 19 March 2017).

Miniyu ASMR (2016) Still Waiting (Role play). https://www.youtube.com/watch?v=cDCE9w-06ml (retrieved 19 March 2017).

Neil Cooper (2017) YouTube channel. https://www.youtube.com/channel/UC6zQkfAvlUBu zGFGYGTfKPA (retrieved 19 March 2017).
Novella, S. (2012) ASMR. NEUROLOGICAblog. http://theness.com/neurologicablog/index.php/asmr (retrieved 19 March 2017).

Phoenician Sailor (2014) YouTube channel. https://www.youtube.com/channel/UCKKaOoUZAR UeHArVEN59GPA (retrieved 19 March 2017).

PixelWhipt (2015) The K3YS: A 360 Degree ASMR Experience. https://littlstar.com/videos/6d352a4b (retrieved 19 March 2017).

Richards, C (2017) History of ASMR. https://asmruniversity.com/history-of-asmr/ (retrieved 19 March 2017).

Smith, S. D., Fredborg, B. K., and Kornelsen, J. (2016) An examination of the default mode network in individuals with autonomous sensory meridian response (ASMR). Social Neuroscience. DOI: $\underline{10.1080 / 17470919.2016 .1188851}$

Stott, K. (2010) A paradoxical relationship. Mental Health Practice, 13(7), pp.15-17.

Taylor Robbins (2016) YouTube channel. https://www.youtube.com/channel/UC8JFV1ICqAlx kg2WW-p3xDQ/feed (retrieved 20 March 2017).

The French Whisperer (2013) YouTube channel. https://www.youtube.com/channel/UCSkS5vtp5gY3 huyVkX4IfMw/featured (retrieved 19 March 2017).

TirarADeguello (2017) Illuminati Infinity Time Loop ASMR.

https://www.youtube.com/watch?v=4ePOD8EJKEw (retrieved 19 March 2017).

Tony Bomboni ASMR (2016) How I Set Up to Make ASMR Videos | Equipment, Tutorial. https://www.youtube.com/watch?v=Xh25fiJfQLA (retrieved 19 March 2017).

Vobis ASMR (2016) The Dreamcatcher. https://www.youtube.com/watch?v=EVDRkjSvwmg (retrieved 19 March 2017).

WhisperingLife (2009) Whisper 1 - hello! https://www.youtube.com/watch?v=lHtgPbfTgKc (retrieved 19 March 2017).

WhispersRed ASMR (2015) HSP Examination Sunshine Academy \#2 - ASMR Role Play | Ear Therapy | Reiki Healing.

https://www.youtube.com/watch?v=mlzvZFKCsn8 (retrieved 23 May 2017). 